Research Article; Received: December 23, 2020; Accepted: August 18, 2021

\title{
GENERALIZED OSCULATING CURVES OF TYPE (n-3) IN THE n-DIMENSIONAL EUCLIDEAN SPACE
}

\author{
Özcan BEKTAŞ and Zafer BEKIRYAZICI
}

Department of Mathematics, Faculty of Arts and Sciences, Recep Tayyip Erdogan University, Rize, TURKEY

\begin{abstract}
In this paper, we give a generalization of the osculating curves to the $n$-dimensional Euclidean space. Based on the definition of an osculating curve in the 3 and 4 dimensional Euclidean spaces, a new type of osculating curve has been defined such that the curve is independent of the $(n-3)$ th binormal vector in the $n$-dimensional Euclidean space, which has been called "a generalized osculating curve of type $(n-3)$ ". We find the relationship between the curvatures for any unit speed curve to be congruent to this osculating curve in $E^{n}$. In particular, we characterize the osculating curves in $E^{n}$ in terms of their curvature functions. Finally, we show that the ratio of the $(n-1)$ th and $(n-2)$ th curvatures of the osculating curve is the solution of an $(n-2)$ th order linear nonhomogeneous differential equation.
\end{abstract}

\section{INTRODUCTION}

Curve theory is a popular research interest in classical differential geometry and osculating curves are a known example in this field. There are many studies on osculating curves in the Euclidean 3 -space $E^{3}$. The significant property of these curves is that the position vector of osculating curves always lie in their osculating planes. The osculating curve $\alpha: I \rightarrow E^{3}$ is defined by

$$
\alpha(s)=\lambda(s) T(s)+\mu(s) N(s),
$$

for some differentiable functions $\lambda$ and $\mu$ of $s \in I \subset \mathbb{R}$, where $T(s)$ is the tangent vector field and $N(s)$ is the normal vector field. Similar curves are present in curve theory such as normal curves, where the position vector always lies in the normal plane, and the rectifying curves, where the position vector always lies in the

2020 Mathematics Subject Classification. Primary 53A04; Secondary 53A07, 34A05.

Keywords. Osculating curve, curvatures, unit speed curve, higher order linear differential equation.

■ozcan.bektas@erdogan.edu.tr; zafer.bekiryazici@erdogan.edu.tr-Corresponding author

(D) 0000-0002-2483-1939; 0000-0001-5671-9995. 
rectifying plane [1]. B. Y. Chen has studied rectifying curves in his paper "When does the position vector of a space curve always lie in its rectifying plane?". Since this study, normal, rectifying and osculating curves have been studied from different perspectives. Some of the studies in the literature have been listed below.

Chen and Dillen have studied rectifying curves in [2], where they established a relationship between rectifying curves and centrodes in mechanics. They also show that rectifying curves satisfy the equality case of a general inequality in their study. The characterization of the rectifying curve in Euclidean 4-space and Minkowski 3-space are given in [3], [4] and [5]. Cambie et al. investigated rectifying curves in an arbitrary dimensional Euclidean space using conditions on their curvature [6]. Additionally, there are some papers on spacelike, timelike and null normal curves in Minkowski space [7], [8]. Characterizations of an osculating curve in the 3 -dimensional Euclidean space has been given in [9] and a specific osculating curve has been defined in the Euclidean 4-space. Normal, osculating and rectifying curves have been defined in the Euclidean and semi Euclidean space by using quaternion algebra in [10], [11], [16] and 25]. Bi-null curves of these types have also been analyzed in $\mathbb{R}_{3}^{6}$ and $\mathbb{R}_{2}^{5}$ in $[12,13]$. Several studies in the literature on the topic of interest of this study can be found in [14-18, 26, 27].

In this paper, using similar methods to those used in [6] and the definition of an osculating curve as stated in [9], we investigate the properties of a generalized form of osculating curves in the $n$-dimensional Euclidean space which are independent of the $(n-3)$ th binormal vector. We call this osculating curve "a generalized osculating curve of type $(n-3)$ ". Firstly, basic concepts of curve theory in $E^{n}$ are given as preliminaries. Then, the characterization of the osculating curves is given in $E^{n}$. The necessary and sufficient condition for a curve to be an osculating curve in the $\mathbf{n}$-dimensional Euclidean space is also obtained. Additionally, using this necessary and sufficient condition, we show that if a curve is an osculating curve in the $\mathbf{n}$-dimensional Euclidean space, its curvatures define a differential equation. Finally, we state the existence and uniqueness of the solution of this differential equation and propose a general form for the general solution of the equation.

\section{Preliminaries}

Basic concepts of curve theory in the $n$-dimensional Euclidean space $E^{n}$ are given in this section. Let $\alpha: I \subset \mathbb{R} \rightarrow E^{n}, s \in I \rightarrow \alpha(s)$ be an arclength parameterized, $n$ times continuously differentiable curve. The curve $\alpha$ is called a unit speed curve if $\langle\alpha, \alpha\rangle=1$, where $\langle$,$\rangle is the function that shows the standart inner product in$ the $n$-dimensional Euclidean space $E^{n}$ given by

$$
\langle X, Y\rangle=\sum_{i=1}^{n} x_{i} y_{i}
$$

for each $X=\left(x_{1}, x_{2}, \ldots, x_{n}\right)$ and $Y=\left(y_{1}, y_{2}, \ldots, y_{n}\right) \in E^{n}$. The norm of $X$ is given by $\|X\|=\sqrt{\langle X, X\rangle}$. On the other hand, if $\|X\|=1$, then $X$ is an unit vector. 
Also, if the curve $\alpha$ in $E^{n}$ is an arclength parameterized curve, then $\left\|\frac{d \alpha}{d s}\right\|=1$. The Serret Frenet formulas for $E^{n}$ are given as the following equations (see [19]):

$$
\begin{aligned}
& T^{\prime}(s)=\kappa_{1}(s) N(s) \\
& N^{\prime}(s)=-\kappa_{1}(s) T(s)+\kappa_{2}(s) B_{1}(s) \\
& B_{1}^{\prime}(s)=-\kappa_{2}(s) N(s)+\kappa_{3}(s) B_{2}(s) \\
& B_{i}^{\prime}(s)=-\kappa_{i+1}(s) B_{i-1}(s)+\kappa_{i+2}(s) B_{i+1}(s), 2 \leq i \leq n-3 \\
& B_{n-2}^{\prime}(s)=-\kappa_{n-1}(s) B_{n-3}(s),
\end{aligned}
$$

where $\kappa_{1}, \kappa_{2}, \kappa_{3}, \ldots, \kappa_{n-1}$ are the curvature functions of the curve and are positive. For more information on curve theory, the reader is advised to see the liteature $[20-22]$.

\section{Osculating curves of type (n-3) in the $\mathbf{n}$-dimensional Euclidean space}

In this section, generalizations of several fundamental definitions, theorems, and results to generalized osculating curves of type (n-3) in the $n$-dimensional Euclidean space are given. All of the mentions to osculating curves in our study refer to the generalized osculating curves of type (n-3) from this point.

Definition 1. Let $\alpha: I \subset \mathbb{R} \rightarrow E^{n}, s \in I \rightarrow \alpha(s)$ be an arclength parameterized, $n$ times continuously differentiable curve. In $E^{n}$, a curve for which the position vector always lies in the orthogonal complement $B_{n-3}^{\perp}(s)$ of its $(n-3)$ th binormal vector field $B_{n-3}(s)$ is called the osculating curve. $B_{n-3}^{\perp}(s)$ is defined as

$$
B_{n-3}^{\perp}(s)=\left\{W \in E^{n} \mid\left\langle W, B_{n-3}(s)\right\rangle=0\right\}
$$

where $\langle$,$\rangle denotes the standard scalar product in E^{n}$. Thus $B_{n-3}^{\perp}(s)$ is a $(n-1)$ dimensional subspace of $E^{n}$, spanned by the tangent, the principal normal, the first binormal, second binormal,..., $(n-4)$ th binormal and, $(n-2)$ th binormal vector fields $T, N, B_{1}(s), \ldots, B_{n-4}(s), B_{n-2}(s)$ respectively. Therefore, the position vector of an osculating curve with respect to a specific origin is given as

$$
\alpha(s)=\mu_{1}(s) T(s)+\mu_{2}(s) N(s)+\sum_{i=1}^{n-4} \mu_{i+2}(s) B_{i}(s)+\mu_{n-1}(s) B_{n-2}(s)
$$

for some differentiable functions $\mu_{i}(1 \leq i \leq n-3)$ of $s \in I \subset \mathbb{R}$.

Theorem 1. Let $\alpha(s)$ be a unit speed curve in $E^{n}$ with nonzero curvatures. Then $\alpha(s)$ is congruent to a osculating curve in $E^{n}$ if and only if

$$
\sum_{z=0}^{n-3}\left(\Gamma_{i, z}(s) \frac{d^{z}}{d s^{z}}\left(\frac{\kappa_{n-1}(s)}{\kappa_{n-2}(s)}\right)\right)^{\prime}-\kappa_{1}(s) \sum_{z=0}^{n-4} \Gamma_{i, z}(s) \frac{d^{z}}{d s^{z}}\left(\frac{\kappa_{n-1}(s)}{\kappa_{n-2}(s)}\right)=\frac{1}{c},
$$

$c \in \mathbb{R}-\{0\}$, where $1 \leq i \leq n-1$. 
Proof. Let $\alpha$ be an arclength parameterized osculating curve in the $n$-dimensional Euclidean space. The derivative of (2) with respect to $s$ for both sides of the equation is

$$
\begin{aligned}
\alpha^{\prime}(s)= & \mu_{1}^{\prime}(s) T(s)+\mu_{1}(s) T^{\prime}(s)+\mu_{2}^{\prime}(s) N(s)+\mu_{2}(s) N^{\prime}(s) \\
& +\sum_{i=2}^{n-4}\left(\mu_{i+2}^{\prime}(s) B_{i}(s)+\mu_{i+2}(s) B_{i}^{\prime}(s)\right) \\
& +\mu_{n-1}^{\prime}(s) B_{n-2}(s)+\mu_{n-1}(s) B_{n-2}(s) .
\end{aligned}
$$

Implementing the Serret Frenet formulas for the $n$-dimensional Euclidean space and rearranging the terms of the right hand side, we get

$$
\begin{aligned}
T(s)= & \left(\mu_{1}^{\prime}(s)-\mu_{2}(s) \kappa_{1}(s)\right) T(s) \\
& +\left(\mu_{1}(s) \kappa_{1}(s)+\mu_{2}^{\prime}(s)-\mu_{3}(s) \kappa_{2}(s)\right) N(s) \\
& +\left(\mu_{2}(s) \kappa_{2}(s)+\mu_{3}^{\prime}(s)-\mu_{4}(s) \kappa_{3}(s)\right) B_{1}(s) \\
& +\sum_{i=2}^{n-5}\left(\mu_{i+1}(s) \kappa_{i+1}(s)+\mu_{i+2}^{\prime}(s)-\mu_{i+3}(s) \kappa_{i+2}(s)\right) B_{i}(s) \\
& +\left(\mu_{n-3}(s) \kappa_{n-3}(s)+\mu_{n-2}^{\prime}(s)\right) B_{n-4}(s) \\
& +\left(\mu_{n-2}(s) \kappa_{n-2}(s)-\mu_{n-1}(s) \kappa_{n-1}(s)\right) B_{n-3}(s) \\
& +\mu_{n-1}^{\prime}(s) B_{n-2}(s)
\end{aligned}
$$

Using the equality of both sides, we get the following expressions for the coefficients of $T(s), N(s), B_{i}(s)$ for $i=2,3, \ldots, n-2$ :

$$
\begin{gathered}
\mu_{1}^{\prime}(s)-\mu_{2}(s) \kappa_{1}(s)=1 \\
\mu_{1}(s) \kappa_{1}(s)+\mu_{2}^{\prime}(s)-\mu_{3}(s) \kappa_{2}(s)=0 \\
\mu_{2}(s) \kappa_{2}(s)+\mu_{3}^{\prime}(s)-\mu_{4}(s) \kappa_{3}(s)=0 \\
\mu_{i+1}(s) \kappa_{i+1}(s)+\mu_{i+2}^{\prime}(s)-\mu_{i+3}(s) \kappa_{i+2}(s)=0,2 \leq i \leq n-3 \\
\mu_{n-3}(s) \kappa_{n-3}(s)+\mu_{n-2}^{\prime}(s)=0 \\
\mu_{n-2}(s) \kappa_{n-2}(s)-\mu_{n-1}(s) \kappa_{n-1}(s)=0 \\
\mu_{n-1}^{\prime}(s)=0 .
\end{gathered}
$$

Starting from (9), we integrate these expressions with respect to $s$ to obtain the coefficient functions

$$
\mu_{n-1}(s)=c, c \in \mathbb{R}
$$


Similarly, the integrations of (7) and (8) yield

$$
\mu_{n-2}(s)=-\frac{\kappa_{n-1}(s)}{\kappa_{n-2}(s)}
$$

and

$$
\mu_{n-3}(s)=-\frac{c}{\kappa_{n-3}(s)}\left(\frac{\kappa_{n-1}(s)}{\kappa_{n-2}(s)}\right)^{\prime} .
$$

On the other hand, for $i=n-4$, and $n-5$, we get the following equations:

$$
\begin{aligned}
\mu_{n-4}(s)= & -c \frac{\kappa_{n-3}(s)}{\kappa_{n-4}(s)}\left(\frac{\kappa_{n-1}(s)}{\kappa_{n-2}(s)}\right)^{\prime} \\
& +\frac{c}{\kappa_{n-4}(s)}\left(\frac{1}{\kappa_{n-3}(s)}\right)^{\prime}\left(\frac{\kappa_{n-1}(s)}{\kappa_{n-2}(s)}\right)^{\prime} \\
& +\frac{c}{\kappa_{n-4}(s)}\left(\frac{1}{\kappa_{n-3}(s)}\right)\left(\frac{\kappa_{n-1}(s)}{\kappa_{n-2}(s)}\right)^{\prime \prime}
\end{aligned}
$$

and

$$
\begin{aligned}
\mu_{n-5}(s)= & -\frac{c}{\kappa_{n-5}(s)}\left(\frac{\kappa_{n-3}(s)}{\kappa_{n-4}(s)}\right)^{\prime}\left(\frac{\kappa_{n-1}(s)}{\kappa_{n-2}(s)}\right) \\
& -\left[\frac{c}{\kappa_{n-5}(s)}\left(\left(\frac{\kappa_{n-3}(s)}{\kappa_{n-4}(s)}\right)-\frac{\kappa_{n-4}(s)}{\kappa_{n-3}(s)}\right)\right]\left(\frac{\kappa_{n-1}(s)}{\kappa_{n-2}(s)}\right)^{\prime} \\
& +\frac{c}{\kappa_{n-5}(s)}\left(\frac{1}{\kappa_{n-4}(s)}\left(\frac{1}{\kappa_{n-3}(s)}\right)^{\prime}\right)^{\prime}\left(\frac{\kappa_{n-1}(s)}{\kappa_{n-2}(s)}\right)^{\prime} \\
& +\frac{2 c}{\kappa_{n-5}(s)} \frac{1}{\kappa_{n-4}(s)} \frac{1}{\kappa_{n-3}(s)}\left(\frac{\kappa_{n-1}(s)}{\kappa_{n-2}(s)}\right)^{\prime \prime} \\
& -\frac{c}{\kappa_{n-5}(s)} \frac{1}{\kappa_{n-4}(s)} \frac{1}{\kappa_{n-3}(s)}\left(\frac{\kappa_{n-1}(s)}{\kappa_{n-2}(s)}\right)^{\prime \prime \prime} .
\end{aligned}
$$

The other curvature functions have expressions of a complicated structure. Hence, we define the following functions to express these curvatures: The function $\Gamma_{n-4,0}(s)$ is defined as

$$
\begin{aligned}
& \Gamma_{n-4,0}(s)=-\frac{\kappa_{n-3}(s)}{\kappa_{n-4}(s)}, \Gamma_{n-4,1}(s)=\frac{1}{\kappa_{n-4}(s)}\left(\frac{1}{\kappa_{n-3}(s)}\right)^{\prime}, \\
& \Gamma_{n-4,2}(s)=\frac{1}{\kappa_{n-4}(s)}\left(\frac{1}{\kappa_{n-3}(s)}\right),
\end{aligned}
$$


then we get

$$
\begin{aligned}
\mu_{n-4}(s)= & c \Gamma_{n-4,0}(s)\left(\frac{\kappa_{n-1}(s)}{\kappa_{n-2}(s)}\right)+c \Gamma_{n-4,1}(s)\left(\frac{\kappa_{n-1}(s)}{\kappa_{n-2}(s)}\right)^{\prime} \\
& +c \Gamma_{n-4,2}(s)\left(\frac{\kappa_{n-1}(s)}{\kappa_{n-2}(s)}\right)^{\prime \prime} .
\end{aligned}
$$

Similarly, $\Gamma_{n-5,0}(s), \Gamma_{n-5,1}(s), \Gamma_{n-5,2}(s)$, and $\Gamma_{n-5,3}(s)$ are defined as

$$
\begin{aligned}
\Gamma_{n-5,0}(s)= & -\frac{1}{\kappa_{n-5}(s)}\left(\frac{\kappa_{n-3}(s)}{\kappa_{n-4}(s)}\right)^{\prime} \\
\Gamma_{n-5,1}(s)= & {\left[\frac{1}{\kappa_{n-5}(s)}\left(\left(\frac{\kappa_{n-3}(s)}{\kappa_{n-4}(s)}\right)-\frac{\kappa_{n-4}(s)}{\kappa_{n-3}(s)}\right)\right] } \\
& +\frac{1}{\kappa_{n-5}(s)}\left(\frac{1}{\kappa_{n-4}(s)}\left(\frac{1}{\kappa_{n-3}(s)}\right)^{\prime}\right)^{\prime}, \\
\Gamma_{n-5,2}(s)= & \frac{2}{\kappa_{n-5}(s)} \frac{1}{\kappa_{n-4}(s)} \frac{1}{\kappa_{n-3}(s)}, \\
\Gamma_{n-5,3}(s)= & -\frac{1}{\kappa_{n-5}(s)} \frac{1}{\kappa_{n-4}(s)} \frac{1}{\kappa_{n-3}(s)},
\end{aligned}
$$

then we get

$$
\begin{aligned}
\mu_{n-5}(s)= & c \Gamma_{n-5,0}(s)\left(\frac{\kappa_{n-1}(s)}{\kappa_{n-2}(s)}\right)+c \Gamma_{n-5,1}(s)\left(\frac{\kappa_{n-1}(s)}{\kappa_{n-2}(s)}\right)^{\prime} \\
& +c \Gamma_{n-5,2}(s)\left(\frac{\kappa_{n-1}(s)}{\kappa_{n-2}(s)}\right)^{\prime \prime}+c \Gamma_{n-5,3}(s)\left(\frac{\kappa_{n-1}(s)}{\kappa_{n-2}(s)}\right)^{\prime \prime \prime} .
\end{aligned}
$$

Altogether, the following expression can be defined for the functions defined above:

$$
\mu_{i}(s)=\sum_{z=0}^{n-i-2} c \Gamma_{i, z}(s) \frac{d^{z}}{d s^{z}}\left(\frac{\kappa_{n-1}(s)}{\kappa_{n-2}(s)}\right), 1 \leq i \leq n-1 .
$$

Thus we get the following coefficient functions for $i=1$, and $i=2$

$$
\mu_{1}(s)=\sum_{z=0}^{n-3} c \Gamma_{i, z}(s) \frac{d^{z}}{d s^{z}}\left(\frac{\kappa_{n-1}(s)}{\kappa_{n-2}(s)}\right)
$$

and

$$
\mu_{2}(s)=\sum_{z=0}^{n-4} c \Gamma_{i, z}(s) \frac{d^{z}}{d s^{z}}\left(\frac{\kappa_{n-1}(s)}{\kappa_{n-2}(s)}\right)
$$


Substituting (16) and (17) into (3), we obtain the relations below

$$
\sum_{z=0}^{n-3}\left(\Gamma_{i, z}(s) \frac{d^{z}}{d s^{z}}\left(\frac{\kappa_{n-1}(s)}{\kappa_{n-2}(s)}\right)\right)^{\prime}-\kappa_{1}(s) \sum_{l=0}^{n-4} \Gamma_{i, z}(s) \frac{d^{z}}{d s^{z}}\left(\frac{\kappa_{n-1}(s)}{\kappa_{n-2}(s)}\right)=\frac{1}{c},
$$

for $c \in \mathbb{R}-0$.

Conversely, consider an arbitrary unit speed curve in $E^{n}$ for which the curvature functions satisfy the relation (18). Then, we consider the the vector $X \in E^{n}$ defined by

$$
\begin{aligned}
X(s)= & \alpha(s)+\sum_{z=0}^{n-3} c \Gamma_{i, z}(s) \frac{d^{z}}{d s^{z}}\left(\frac{\kappa_{n-1}(s)}{\kappa_{n-2}(s)}\right) T(s) \\
& +\sum_{z=0}^{n-4} c \Gamma_{i, z}(s) \frac{d^{z}}{d s^{z}}\left(\frac{\kappa_{n-1}(s)}{\kappa_{n-2}(s)}\right) N(s) \\
& +\ldots+\sum_{z=0}^{n-i-4} c \Gamma_{i, z}(s) \frac{d^{z}}{d s^{z}}\left(\frac{\kappa_{n-1}(s)}{\kappa_{n-2}(s)}\right) B_{i}(s) \\
& +\ldots-\left(\frac{c}{\kappa_{n-3}(s)}\left(\frac{\kappa_{n-1}(s)}{\kappa_{n-2}(s)}\right)\right) B_{n-5}(s) \\
& -\left(\frac{\kappa_{n-1}(s)}{\kappa_{n-2}(s)}\right) B_{n-4}(s) \\
& +c B_{n-2}(s)
\end{aligned}
$$

It can be seen that $X^{\prime}(s)=0$ through the relations (1) and (18). Thus, $X$ is a constant vector. This implies that $\alpha$ is congruent to an osculating curve. Hence, the proof is complete.

Theorem 2. Let $\alpha(s)$ be a unit speed osculating curve in $E^{n}$ with nonzero curvatures. Then the following hold:

i) The tangential, the principal normal, the first, the second, ..., the $i$-th,..., the $(n-5)$ th, and $(n-4)$ th binormal components of the position vector of the curve are respectively given by

$$
\begin{aligned}
\langle\alpha(s), T(s)\rangle & =\sum_{z=0}^{n-3} c \Gamma_{i, z}(s) \frac{d^{z}}{d s^{z}}\left(\frac{\kappa_{n-1}(s)}{\kappa_{n-2}(s)}\right), \\
\langle\alpha(s), N(s)\rangle & =\sum_{z=0}^{n-4} c \Gamma_{i, z}(s) \frac{d^{z}}{d s^{z}}\left(\frac{\kappa_{n-1}(s)}{\kappa_{n-2}(s)}\right) . \\
\left\langle\alpha(s), B_{1}(s)\right\rangle & =\sum_{z=0}^{n-5} c \Gamma_{i, z}(s) \frac{d^{z}}{d s^{z}}\left(\frac{\kappa_{n-1}(s)}{\kappa_{n-2}(s)}\right),
\end{aligned}
$$




$$
\begin{gathered}
\left\langle\alpha(s), B_{2}(s)\right\rangle=\sum_{z=0}^{n-6} c \Gamma_{i, z}(s) \frac{d^{z}}{d s^{z}}\left(\frac{\kappa_{n-1}(s)}{\kappa_{n-2}(s)}\right), \\
\vdots \\
\left\langle\alpha(s), B_{i}(s)\right\rangle=\sum_{z=0}^{n-i-4} c \Gamma_{i, z}(s) \frac{d^{z}}{d s^{z}}\left(\frac{\kappa_{n-1}(s)}{\kappa_{n-2}(s)}\right) \\
\vdots \\
\left\langle\alpha(s), B_{n-5}(s)\right\rangle=-\frac{c}{\kappa_{n-3}(s)}\left(\frac{\kappa_{n-1}(s)}{\kappa_{n-2}(s)}\right)^{\prime}, \\
\left\langle\alpha(s), B_{n-4}(s)\right\rangle=-\frac{\kappa_{n-1}(s)}{\kappa_{n-2}(s)} .
\end{gathered}
$$

ii) The $(n-2)$ th binormal component of the position vector of the curve is a nonzero constant.

Conversely, if $\alpha(s)$ is a unit speed curve in $E^{n}$ with non-zero curvatures and one of the statements (i), (ii) holds, then $\alpha(s)$ is an osculating curve or is congruent to an osculating curve in $E^{n}$.

Proof. By using the relations (2) and (3)-(9), the position vector of the curve can be written as follows:

$$
\begin{aligned}
\alpha(s)= & \sum_{z=0}^{n-3} c \Gamma_{i, z}(s) \frac{d^{z}}{d s^{z}}\left(\frac{\kappa_{n-1}(s)}{\kappa_{n-2}(s)}\right) T(s) \\
& +\sum_{z=0}^{n-4} c \Gamma_{i, z}(s) \frac{d^{z}}{d s^{z}}\left(\frac{\kappa_{n-1}(s)}{\kappa_{n-2}(s)}\right) N(s) \\
& +\ldots+\sum_{z=0}^{n-i-4} c \Gamma_{i, z}(s) \frac{d^{z}}{d s^{z}}\left(\frac{\kappa_{n-1}(s)}{\kappa_{n-2}(s)}\right) B_{i}(s) \\
& +\ldots-\left(\frac{c}{\kappa_{n-3}(s)}\left(\frac{\kappa_{n-1}(s)}{\kappa_{n-2}(s)}\right)^{\prime}\right) B_{n-5}(s) \\
& -\left(\frac{\kappa_{n-1}(s)}{\kappa_{n-2}(s)}\right) B_{n-4}(s)+c B_{n-2}(s)
\end{aligned}
$$

From (19), we get (19)- (25). Thus, (i) and (ii) have been proved.

Conversely, assume that statements (i) and (ii) hold. By taking the derivative of $\left\langle\alpha(s), B_{n-4}(s)\right\rangle=-\frac{\kappa_{n-1}(s)}{\kappa_{n-2}(s)}$ with respect to $s$ and using (1) we get,

$$
-\kappa_{n-3}(s)\left\langle\alpha(s), B_{n-5}(s)\right\rangle+\kappa_{n-2}(s)\left\langle\alpha(s), B_{n-3}(s)\right\rangle=-\frac{\kappa_{n-1}(s)}{\kappa_{n-2}(s)} .
$$


By using $\left\langle\alpha(s), B_{n-5}(s)\right\rangle=-\frac{c}{\kappa_{n-3}(s)}\left(\frac{\kappa_{n-1}(s)}{\kappa_{n-2}(s)}\right)^{\prime}$ and $\kappa_{n-2}(s) \neq 0$, we get $\left\langle\alpha(s), B_{n-3}(s)\right\rangle=0$, which means that this is an osculating curve.

If statement (ii) holds, then we have $\left\langle\alpha(s), B_{n-2}(s)\right\rangle=c, c \in \mathbb{R}-\{0\}$. Differentiating the previous equation with respect to $s$ and using (1), we find $-\kappa_{n-1}(s)\left\langle\alpha(s), B_{n-3}(s)\right\rangle=0$. It follows that $\left\langle\alpha(s), B_{n-3}(s)\right\rangle=0$ and hence the curve $\alpha$ is an osculating curve.

Theorem 3. Let $\alpha(s)$ be a unit speed osculating curve in $E^{n}$ with nonzero curvatures. The differential equation

$$
\sum_{z=0}^{n-3}\left(\Gamma_{i, z}(s) \frac{d^{z}}{d s^{z}}\left(\frac{\kappa_{n-1}(s)}{\kappa_{n-2}(s)}\right)\right)^{\prime}-\kappa_{1}(s) \sum_{z=0}^{n-4} \Gamma_{i, z}(s) \frac{d^{z}}{d s^{z}}\left(\frac{\kappa_{n-1}(s)}{\kappa_{n-2}(s)}\right)=\frac{1}{c},
$$

where $c \in \mathbb{R}-\{0\} ., n>4, i=1,2, \ldots, n-1$ with the initial conditions

$$
\frac{\kappa_{n-1}\left(s_{0}\right)}{\kappa_{n-2}\left(s_{0}\right)}=k_{0},\left[\frac{\kappa_{n-1}\left(s_{0}\right)}{\kappa_{n-2}\left(s_{0}\right)}\right]^{\prime}=k_{1}, \ldots,\left[\frac{\kappa_{n-1}\left(s_{0}\right)}{\kappa_{n-2}\left(s_{0}\right)}\right]^{(n-3)}=k_{(n-3)}
$$

for $s_{0} \in I \subset \mathbb{R}$ has a unique solution on an open interval $I \subset \mathbb{R}$ if the functions

$$
\begin{aligned}
& {\left[\Gamma_{i, 0}^{\prime}(s)-\kappa_{1} \Gamma_{i, 0}(s)\right],\left[\Gamma_{i, 0}(s)+\Gamma_{i, 1}^{\prime}(s)-\kappa_{1} \Gamma_{i, 1}(s)\right], \ldots,} \\
& {\left[\Gamma_{i, m-1}(s)+\Gamma_{i, m}^{\prime}(s)-\kappa_{1} \Gamma_{i, m}(s)\right], \ldots,\left[\Gamma_{i, n-4}(s)+\Gamma_{i, n-3}^{\prime}(s)\right],\left[\Gamma_{i, n-3}(s)\right], \frac{1}{c}}
\end{aligned}
$$

are continuous on $I$ and $\left[\Gamma_{i, 0}^{\prime}(s)-\kappa_{1} \Gamma_{i, 0}(s)\right] \neq 0, \ldots,\left[\Gamma_{i, n-3}(s)\right] \neq 0$ for every $s \in I$. This equation has a general solution of the form

$$
\left(\frac{\kappa_{n-1}(s)}{\kappa_{n-2}(s)}\right)=c_{1}\left(\frac{\kappa_{n-1}}{\kappa_{n-2}}\right)_{1}(s)+\ldots+c_{n-2}\left(\frac{\kappa_{n-1}}{\kappa_{n-2}}\right)_{n-2}(s)+\left(\frac{\kappa_{n-1}}{\kappa_{n-2}}\right)_{p}
$$

where $\left(\frac{\kappa_{n-1}}{\kappa_{n-2}}\right)_{1}(s),\left(\frac{\kappa_{n-1}}{\kappa_{n-2}}\right)_{2}(s), \ldots,\left(\frac{\kappa_{n-1}}{\kappa_{n-2}}\right)_{n-2}(s)$ form the fundamental set of solutions for the homogeneous equation

$$
\begin{aligned}
& \left(\frac{\kappa_{n-1}(s)}{\kappa_{n-2}(s)}\right)^{(n-2)}+\left(\frac{\Gamma_{i, n-4}(s)+\Gamma_{i, n-3}^{\prime}(s)}{\Gamma_{i, n-3}(s)}\right)\left(\frac{\kappa_{n-1}(s)}{\kappa_{n-2}(s)}\right)^{(n-3)}+\ldots \\
& +\left(\frac{\Gamma_{i, m-1}(s)+\Gamma_{i, m}^{\prime}(s)-\kappa_{1}(s) \Gamma_{i, m}(s)}{\Gamma_{i, n-3}(s)}\right)\left(\frac{\kappa_{n-1}(s)}{\kappa_{n-2}(s)}\right)^{(m)}+\ldots \\
& +\left(\frac{\Gamma_{i, 0}(s)-\kappa_{1}(s) \Gamma_{i, 0}(s)}{\Gamma_{i, n-3}(s)}\right)\left(\frac{\kappa_{n-1}(s)}{\kappa_{n-2}(s)}\right)=0 .
\end{aligned}
$$

satisfying the condition

$$
W\left(\left(\frac{\kappa_{n-1}}{\kappa_{n-2}}\right)_{1},\left(\frac{\kappa_{n-1}}{\kappa_{n-2}}\right)_{2}, \ldots,\left(\frac{\kappa_{n-1}}{\kappa_{n-2}}\right)_{n-2}\right)
$$




$$
=\left|\begin{array}{cccc}
\left(\frac{\kappa_{n-1}}{\kappa_{n-2}}\right)_{1} & \left(\frac{\kappa_{n-1}}{\kappa_{n-2}}\right)_{2} & \cdots & \left(\frac{\kappa_{n-1}}{\kappa_{n-2}}\right)_{n-2} \\
\left(\frac{\kappa_{n-1}}{\kappa_{n-2}}\right)_{1}^{\prime} & \left(\frac{\kappa_{n-1}}{\kappa_{n-2}}\right)_{2}^{\prime} & \cdots & \left(\frac{\kappa_{n-1}}{\kappa_{n-2}}\right)_{n-2} \\
\ldots & \cdots & \ldots \\
\left(\frac{\kappa_{n-1}}{\kappa_{n-2}}\right)_{1}^{(n-3)} & \left(\frac{\kappa_{n-1}}{\kappa_{n-2}}\right)_{2}^{(n-3)} & \ldots & \left(\frac{\kappa_{n-1}}{\kappa_{n-2}}\right)_{n-2}^{(n-3)}
\end{array}\right| \neq 0,
$$

$\left(\frac{\kappa_{n-1}}{\kappa_{n-2}}\right)_{p}$ is a particular solution of the initial value problem and $c_{1}, c_{2}, \ldots, c_{n-2}$ are arbitrary constants.

Proof. If the summation operators are expanded in the differential equation, we get

$$
\begin{aligned}
& \left(\Gamma_{i, 0}(s) \frac{\kappa_{n-1}(s)}{\kappa_{n-2}(s)}\right)^{\prime}+\left(\Gamma_{i, 1}(s)\left(\frac{\kappa_{n-1}(s)}{\kappa_{n-2}(s)}\right)^{\prime}\right)^{\prime}+\left(\Gamma_{i, 2}(s)\left(\frac{\kappa_{n-1}(s)}{\kappa_{n-2}(s)}\right)^{\prime \prime}\right) \\
& +\left(\Gamma_{i, 3}(s)\left(\frac{\kappa_{n-1}(s)}{\kappa_{n-2}(s)}\right)^{\prime \prime \prime}\right)^{\prime}+\ldots+\left(\Gamma_{i, n-5}(s)\left(\frac{\kappa_{n-1}(s)}{\kappa_{n-2}(s)}\right)^{(n-5)}\right)^{\prime} \\
& +\left(\Gamma_{i, n-4}(s)\left(\frac{\kappa_{n-1}(s)}{\kappa_{n-2}(s)}\right)^{(n-4)}\right)^{\prime}+\left(\Gamma_{i, n-3}(s)\left(\frac{\kappa_{n-1}(s)}{\kappa_{n-2}(s)}\right)^{(n-3)}\right)^{\prime} \\
& -\kappa_{1}\left[\Gamma_{i, 0}(s)\left(\frac{\kappa_{n-1}(s)}{\kappa_{n-2}(s)}\right)+\ldots+\Gamma_{i, n-4}(s)\left(\frac{\kappa_{n-1}(s)}{\kappa_{n-2}(s)}\right)^{(n-4)}\right]=\frac{1}{c} .
\end{aligned}
$$

Applying the derivations in the first summation and collecting the derivatives of same order yields

$$
\begin{aligned}
& {\left[\Gamma_{i, 0}^{\prime}(s)-\kappa_{1} \Gamma_{i, 0}(s)\right]\left(\frac{\kappa_{n-1}(s)}{\kappa_{n-2}(s)}\right)+\left[\Gamma_{i, 0}(s)+\Gamma_{i, 1}^{\prime}(s)-\kappa_{1} \Gamma_{i, 1}(s)\right]\left(\frac{\kappa_{n-1}(s)}{\kappa_{n-2}(s)}\right)^{\prime}} \\
& +\left[\Gamma_{i, 1}(s)+\Gamma_{i, 2}^{\prime}(s)-\kappa_{1} \Gamma_{i, 2}(s)\right]\left(\frac{\kappa_{n-1}(s)}{\kappa_{n-2}(s)}\right)^{\prime \prime}+\ldots \\
& +\left[\Gamma_{i, n-5}(s)+\Gamma_{i, n-4}^{\prime}(s)-\kappa_{1} \Gamma_{i, n-4}(s)\right]\left(\frac{\kappa_{n-1}(s)}{\kappa_{n-2}(s)}\right)^{(n-4)} \\
& +\left[\Gamma_{i, n-4}(s)+\Gamma_{i, n-3}^{\prime}(s)\right]\left(\frac{\kappa_{n-1}(s)}{\kappa_{n-2}(s)}\right)^{(n-3)}+\Gamma_{i, n-3}(s)\left(\frac{\kappa_{n-1}(s)}{\kappa_{n-2}(s)}\right)^{(n-2)}=\frac{1}{c}
\end{aligned}
$$

This equation is a nonhomogeneous linear differential equation of the order $n-$ 2. Considered along with the initial conditions, $\frac{\kappa_{n-1}\left(s_{0}\right)}{\kappa_{n-2}\left(s_{0}\right)}=k_{0},\left[\frac{\kappa_{n-1}\left(s_{0}\right)}{\kappa_{n-2}\left(s_{0}\right)}\right]^{\prime}=$ $k_{1}, \ldots,\left[\frac{\kappa_{n-1}\left(s_{0}\right)}{\kappa_{n-2}\left(s_{0}\right)}\right]^{(n-3)}=k_{n-3}$, it defines an initial value problem. The continuity of the coefficients of the higher order linear differential equation $\left[\Gamma_{i, 0}^{\prime}(s)-\right.$ $\left.\kappa_{1} \Gamma_{i, 0}(s)\right],\left[\Gamma_{i, 0}(s)+\Gamma_{i, 1}^{\prime}(s)-\kappa_{1} \Gamma_{i, 1}(s)\right], \ldots,\left[\Gamma_{i, m-1}(s)+\Gamma_{i, m}^{\prime}(s)-\kappa_{1} \Gamma_{i, m}(s)\right], \ldots$, 
$\left[\Gamma_{i, n-4}(s)+\Gamma_{i, n-3}^{\prime}(s)\right],\left[\Gamma_{i, n-3}(s)\right]$ and the function $\frac{1}{c}$ guarentees the existence and uniqueness of the solution for the initial value problem on $I \subset \mathbb{R}$ since $\left[\Gamma_{i, 0}^{\prime}(s)-\right.$ $\left.\kappa_{1} \Gamma_{i, 0}(s)\right] \neq 0, \ldots,\left[\Gamma_{i, n-3}(s)\right] \neq 0$ for every $s \in I$ [23]. Division of the equation by the coefficient of the highest order derivative gives

$$
\begin{aligned}
& \left(\frac{\kappa_{n-1}(s)}{\kappa_{n-2}(s)}\right)^{(n-2)}+\left[\frac{\Gamma_{i, n-4}(s)+\Gamma_{i, n-3}^{\prime}(s)}{\Gamma_{i, n-3}(s)}\right]\left(\frac{\kappa_{n-1}(s)}{\kappa_{n-2}(s)}\right)^{(n-3)} \\
& +\left[\frac{\Gamma_{i, n-5}(s)+\Gamma_{i, n-4}^{\prime}(s)-\kappa_{1} \Gamma_{i, n-4}(s)}{\Gamma_{i, n-3}(s)}\right]\left(\frac{\kappa_{n-1}(s)}{\kappa_{n-2}(s)}\right)^{(n-4)} \\
& +\left[\frac{\Gamma_{i, 0}(s)+\Gamma_{i, 1}^{\prime}(s)-\kappa_{1} \Gamma_{i, 1}(s)}{\Gamma_{i, n-3}(s)}\right]\left(\frac{\kappa_{n-1}(s)}{\kappa_{n-2}(s)}\right)^{\prime} \\
& +\left[\frac{\Gamma_{i, 0}^{\prime}(s)-\kappa_{1} \Gamma_{i, 0}(s)}{\Gamma_{i, n-3}(s)}\right]\left(\frac{\kappa_{n-1}(s)}{\kappa_{n-2}(s)}\right)=\frac{1}{c \Gamma_{i, n-3}(s)},
\end{aligned}
$$

such that $\Gamma_{i, n-3} \neq 0$. The continuity of the new coefficients comes from the continuity assumption of the theorem and the fact that $\left[\Gamma_{i, n-3}(s)\right] \neq 0$. Hence, The homogeneous version of this linear differential equation has a fundamental set of solutions on $I \subset \mathbb{R}$ containing solutions of the form $\left(\frac{\kappa_{n-1}}{\kappa_{n-2}}\right)_{k}$ for $k=1,2, \ldots 23$. The fundamental set of solutions is linearly independent if and only if

$$
\begin{aligned}
& W\left(\left(\frac{\kappa_{n-1}}{\kappa_{n-2}}\right)_{1},\left(\frac{\kappa_{n-1}}{\kappa_{n-2}}\right)_{2}, \ldots,\left(\frac{\kappa_{n-1}}{\kappa_{n-2}}\right)_{n-2}\right) \\
& =\left|\begin{array}{cccc}
\left(\frac{\kappa_{n-1}}{\kappa_{n-2}}\right)_{1} & \left(\frac{\kappa_{n-1}}{\kappa_{n-2}}\right)_{2} & \ldots & \left(\frac{\kappa_{n-1}}{\kappa_{n-2}}\right)_{n-2} \\
\left(\frac{\kappa_{n-1}}{\kappa_{n-2}}\right)_{1}^{\prime} & \left(\frac{\kappa_{n-1}}{\kappa_{n-2}}\right)_{2}^{\prime} & \ldots & \left(\frac{\kappa_{n-1}}{\kappa_{n-2}}\right)_{n-2}^{\prime} \\
\ldots & \ldots & & \ldots \\
\left(\frac{\kappa_{n-1}}{\kappa_{n-2}}\right)_{1}^{(n-3)} & \left(\frac{\kappa_{n-1}}{\kappa_{n-2}}\right)_{2}^{(n-3)} & \ldots & \left(\frac{\kappa_{n-1}}{\kappa_{n-2}}\right)_{n-2}^{(n-3)}
\end{array}\right| \neq 0
\end{aligned}
$$

for every $s \in I$ and the superposition principle suggests that the homogeneous linear differential equation has a general solution of the form

$$
\left(\frac{\kappa_{n-1}(s)}{\kappa_{n-2}(s)}\right)=c_{1}\left(\frac{\kappa_{n-1}}{\kappa_{n-2}}\right)_{1}(s)+c_{2}\left(\frac{\kappa_{n-1}}{\kappa_{n-2}}\right)_{2}(s)+\ldots+c_{n-2}\left(\frac{\kappa_{n-1}}{\kappa_{n-2}}\right)_{n-2}(s) .
$$

for arbitrary constants $c_{i}, i=1,2, \ldots ., n-2[23]$. Using the initial conditions, the particular solution can be found as

$$
\left(\frac{\kappa_{n-1}(s)}{\kappa_{n-2}(s)}\right)=c_{1}\left(\frac{\kappa_{n-1}}{\kappa_{n-2}}\right)_{1}(s)+\ldots+c_{n-2}\left(\frac{\kappa_{n-1}}{\kappa_{n-2}}\right)_{n-2}(s)+\left(\frac{\kappa_{n-1}}{\kappa_{n-2}}\right)_{p} .
$$


There are several methods in the literature for analyzing the solutions of higher order linear differential equations. For instance, the variation of parameters method proposes a particular solution for the nonhomogeneous differential equation in the form of

$$
\left(\frac{\kappa_{n-1}(s)}{\kappa_{n-2}(s)}\right)=\sum_{m=1}^{n-2}\left(\frac{\kappa_{n-1}}{\kappa_{n-2}}\right)_{m}(s) \int \frac{\left(c \Gamma_{i, n-3}(t)\right)^{-1} W_{m}(t)}{W(t)} d t
$$

where $\left(\frac{\kappa_{n-1}}{\kappa_{n-2}}\right)_{m}(s)$ form the fundamental set of solutions and $W_{m}(t)$ are obtained by replacing the $m$-th column of the Wronskian by $\left(0,0, \ldots,\left(c \Gamma_{i, n-3}(t)\right)^{-1}\right) 24$.

\section{Conclusion}

In this paper, we have investigated some concepts of osculating curves, defined on 3- and 4-dimensional Euclidean spaces, on the $n$-dimensional Euclidean space. This generalization of osculating curves to $E^{n}$ has been called "generalized osculating curve of type $(n-3)$. A total of $n-2$ generalizations of osculating curves to $E^{n}$ can be found by using the other binormal vectors. However, we have used the $(n-3)$ th binormal vector for the generalization since the relations between the curvatures provide meaningful results. Since the differential equation that gives the relation between curvature functions of the osculating curve in the $n$-dimensional Euclidean space is a higher order differential equation, we have invesitaged the existence and uniquness of a general solution for the initial value problem of order $n-2$. The differential equation of order $n-2$ is a linear differential equation with variable coefficients. Several methods in the literature can be used for analyzing the particular solution of the higher order differential equation.

Author Contribution Statements The authors jointly worked on the results and they read and approved the final manuscript.

Declaration of Competing Interests The authors declare that they have no competing interest.

\section{REFERENCES}

[1] Chen, B. Y., When does the position vector of a space curve always lie in its rectifying plane?, The American Mathematical Monthly, 110(2) (2003), 147-152. https://doi.org/10.1080/00029890.2003.11919949

[2] Chen, B. Y., Dillen, F., Rectifying curves as centrodes and extremal curves, Bulletin of the Institute of Mathematics Academia Sinica, 33(2) (2005), 77-90.

[3] Ilarslan, K., Nesovic, E., Petrovic-Torgasev, M., Some characterizations of rectifying curves in the Minkowski 3-space, Novi Sad Journal of Mathematics, 33(2) (2003), 23-32.

[4] Ilarslan, K., Nesovic, E., On rectifying curves as centrodes and extremal curves in the Minkowski 3-space, Novi Sad Journal of Mathematics, 37(1) (2007), 53-64.

[5] Ilarslan, K., Nesovic, E., Some characterizations of rectifying curves in the Euclidean space $E^{4}$, Turkish Journal of Mathematics, 32(1) (2008), 21-30. 
[6] Cambie, S., Goemans, W., Van den Bussche, I., Rectifying curves in the $n$-dimensional Euclidean space, Turkish Journal of Mathematics, 40(1) (2016), 210-223.

[7] Ilarslan, K., Spacelike normal curves in Minkowski space, Turkish Journal of Mathematics $E_{1}^{3}, 29(1)(2005), 53-63$.

[8] Ilarslan, K., Nesovic, E., Timelike and null normal curves in Minkowski space $E_{1}^{3}$, Indian Journal of Pure ES Applied Mathematics, 35(7) (2004), 881-888.

[9] Ilarslan, K., Nesovic, E., Some characterizations of osculating curves in the Euclidean spaces, Demonstratio Mathematica, 41(4) (2008), 931-940. https://doi.org/10.1515/dema-2008-0421

[10] Yildiz, O. G., Ozkaldi Karakus, O., On the quaternionic normal curves in the Euclidean space, 2nd International Eurasian Conference on Mathematical Sciences and Applications, (2013), 26-29.

[11] Yildiz, O. G., Ozkaldi Karakus, O., On the quaternionic normal curves in the semiEuclidean space $E_{2}^{4}$, International Journal of Mathematical Combinatorics, 3 (2016), 68-76. https://doi.org/10.5281/zenodo.825065

[12] Ucum, A., Sakaki, M., Ilarslan, K., On osculating, normal and rectifying bi-null curves in $R_{3}^{6}$, Journal of Dynamical Systems and Geometric Theories, 15(1) (2017), 1-13. https://doi.org/10.1080/1726037X.2017.1323415

[13] Ilarslan, K., Sakaki, M., Ucum, A., On osculating, normal and rectifying binull curves in $R_{2}^{5}$, Novi Sad Journal of Mathematics, 48(1) (2018), 9-20. https://doi.org/10.30755/NSJOM.05268

[14] Ilarslan, K., Kilic, N., Erdem, H. A., Osculating curves in 4-dimensional semi-Euclidean space with index 2, Open Mathematics, 15(1) (2017), 562-567. https://doi.org/10.1515/math-20170050

[15] Kulahci, M., Almaz, F., Some characteristics of osculating curves in the lightlike cone, Boletim da Sociedade Paranaense de Matematica, 35(2) (2017), 39-48. https://doi.org/10.5269/bspm.v35i2.26227

[16] Bektas, O., Gurses, N., Yuce, S., Quaternionic osculating curves in Euclidean and semiEuclidean space, Journal of Dynamical Systems and Geometric Theories, 14(1) (2016), 65-84. https://doi.org/10.1080/1726037X.2016.1177935

[17] Ilarslan, K., Nesovic, E., Some characterizations of null osculating curves in the Minkowski space-time, Proceedings of the Estonian Academy of Sciences, 61(1) (2012), 18. https://doi.org/10.3176/proc.2012.1.01

[18] Ilarslan, K., Nesovic, E., The first kind and the second kind osculating curves in Minkowski space-time, Comptes rendus de l'Academie bulgare des Sciences, 62(6) (2009), 677-686.

[19] Gluck, H., Higher curvatures of curves in Euclidean space, The American Mathematical Monthly, 73(7) (1966), 699-704. https://doi.org/10.2307/2313974

[20] Kuhnel, W., Differential Geometry: Curves-Surfaces-Manifolds, Braunschweig, Wiesbaden, 1999.

[21] O'Neill, B., Semi-Riemannian Geometry with Applications to Relativity, Academic Press, New York, 1983.

[22] Struik, D. J., Lectures on Classical Differential Geometry, Addison-Wesley Press, Reading, Massachusetts, 1950.

[23] Zill, D. G., Wright, W. S., Differential Equations with Boundary-Value Problems, 8th Edition, Cengage Learning, 2012.

[24] Boyce, W. E., DiPrima, R. C., Meade, D. B., Elementary Differential Equations and Boundary Value Problems, Wiley, New York, 1992.

[25] Gungor, M. A., Tosun, M., Some characterizations of quaternionic rectifying curves, Differential Geometry - Dynamical Systems, 13 (2011), 89-100.

[26] Ucum, A., Kecilioglu, O., Ilaslan, K., Generalized Bertrand curves with timelike (1,3)-normal plane in Minkowski space-time, Kuwait Journal of Science, 42(3) (2015), 10-27. 
[27] Ilarslan, K., Nesovic, E., Tensor product surfaces of a Lorentzian space curve and a Euclidean plane curve, Kuwait Journal of Science and Engineering, 34(2A) (2007), 41-55. 